\title{
The Origin of Gravitational and Electric Forces, the Nature of Electromagnetic Waves
}

\author{
Raoul Charreton \\ 104 Quai Louis Blériot, Paris, France \\ Email: raoul.charreton@mines-paris.org \\ Received March 30, 2012; revised May 9, 2012; accepted May 28, 2012
}

\begin{abstract}
We have proposed a prequantum physics, itself founded on classical mechanics completed by the existence of an universal cloud of tiny particles noted $U$. These $U$ particle command the mass, variable, of electron, neutron, proton, and atom particles noted $M$. The "shocks" between $U$ and $M$ particles in the cloud, with screen effect, give birth to electrical forces among charged particles with very small differences between attractive and repulsive forces, and to certain gravitational forces. This cloud with the electromagnetic waves propagating thus recalls an ether, yet much different regarding its effects on the inertial mass of any particle within it. The electromagnetic wave and the photon look like if they were born from a statistical mechanics induced by the universal cloud, and their status, in this regard, may be compared to the status conferred by atomics to a temperature or a pressure. The wave transversality is explained. By the same token, one understands why the photon, a vectorial bearer of a statistical information, may thus describe a particle as well as a wave.
\end{abstract}

Keywords: Prequantum Physics

\section{The Screen Effect}

Be an universal cloud of tiny particles noted $U$ and $M$ a particle like an atom, a proton or an electron inside this cloud. Let us suppose that the $M$ particle is incessantly knocked by $U$ particles and blown like a down by wind. Of course the word "knocked" calls for a few additions to define a precise model of "shocks". We already gave some indications on this matter in a previous note titled "A prequantum physics" [1] and we intend to complete them.

However, we underline an important point, namely that the shocks are not elastic. Each $M$ particle is surrounded by a tight train of particles $U$ which are part of its internal structure and grant it an inertial mass. A $M$ particle with a void train of particles has no mass. At every non-elastic shock, the train of a $M$ particle, and therefore its mass, are modified ${ }^{1}$.

Let us now suppose that, in the cloud, there are two particles $M$ called $M_{1}$ and $M_{2}$ not too far away from each other. The $U$ environment of $M_{1}$ is slightly modified by the presence of $M_{2}$ because of a screen effect, $M_{2}$ inter-

\footnotetext{
${ }^{1} \mathrm{~A}$ train is modified when its number of $U$ particles changes but it can also be modified by a change in the way the $U$ particles are disposed. (For instance, in a train of 8-10 similar particles in contact, particles may either be in a single file specifically oriented, or gathered within a circle in an oriented plan, or at the nodes of an oriented tridimensional netted network, etc.)
}

cepting from time to time a $U$ particle which, absent $M_{2}$, would have knocked $M_{1}$. And vice-versa. Such a screen effect induces, under diverse conditions, an attraction law in squared distance inverse.

Let us choose for $M_{1}$ a proton noted $P$, for $M_{2}$ an electron noted $E$, and then let us adjust the words describing "shock of $U$ over $P$ " and "shock of $U$ over $E$ " in such a way as the induced attraction law be the electrical attraction between a proton and an electron. The universal cloud seems to induce this attraction and this only. In particular, it does not induce any gravitational attraction between $P$ and $E$.

Let us now choose for $M_{1}$ an hydrogen atom noted $A_{1}$, for $M_{2}$ another hydrogen atom noted $A_{2}$, and then let us adjust the words describing "shock of $U$ over any hydrogen atom noted $A$ " in such a way as the induced attraction law be the gravitational attraction law between two not charged hydrogen atoms. The universal cloud seems to induce this attraction and this only. In particular, it does nor induce any electrical attraction between $A_{1}$ and $A_{2}$.

Let us suppose that the two $A_{1}$ and $A_{2}$ particles are sufficiently far away from each other. The particle $A_{1}$ is made of a proton and an electron, the influence of the $A_{2}$ particle over the $A_{1}$ particle is weak because of its remoteness and the successive shocks of $U$ particles on the proton and electron $A_{1}$ progressively command the spati- 
otemporal arrangement of an hydrogen atom in the fundamental state, cf. [1]. The same applies to $A_{2}$. In short, the hydrogen atom would be induced in quantum mechanics by the electromagnetic interaction between a proton and an electron, in prequantum physics by screen effect.

We know arrive to the paradoxical point, how can the screen effect of a couple proton-electron be much smaller than the effect of each of its components? Because the gravitational interaction between two atoms is much smaller, at equal distance, than the electrical one between its atomic components. Defining the circumstances of a shock relies on cross sections, and the paradox is: how can the cross section of an hydrogen atom be much smaller than those of its proton and electron components?

The explanation is not straightforward and we will progressively to emerge.

We note as usual respectively $G$ and $\alpha$, the Newton's law and fine structure constants which rule, $G$ the gravitational attraction, and $\alpha$ the electrical attraction.

Natural units: We retain them for time, length and mass as to make Planck's constant $h=1$ and not $h=2 \pi$, the speed of light in the $U$ universal cloud $c=1$, the average inertial mass of an isolated electron with no relative speed versus the cloud, $m=1$.

The values of these units in the International System are the following:

Natural unit of time $=8.1 E^{-21}$ second.

Natural unit of length $=2.4 E^{-12}$ meter.

Natural unit of mass $=9.1 E^{-31}$ kilogram.

Natural unit of energy $m \cdot c^{2}=8.2 E^{-14}$ Joule or 511 $\mathrm{keV}$ (kilo electron-volt).

\section{Expected Effects Thanks to the Symmetries}

By hypothesis, the universal cloud has certain symmetries:

1) We suppose that the $U$ particles are moving on a straight line and that their free course is large, comparable to the galactic ray.

2) The density of probability of the speed of a $U$ particle be aligned on a direction is the same whatever the direction.

3) The number of $U$ particles present at any time in a spatial volume is a random variable with a Poisson law according to the following parameters.

Be $N$ the set of the positive natural integers $0,1,2$,

Be $\lambda$ a positive real number and $p_{j}(\lambda)=\frac{e^{-\lambda} \cdot \lambda^{j}}{j !}, j \in N$

$N$. The random variable $X$ defined by $P(X=j)=p_{j}(\lambda)$ follows a Poisson's law.

Be $g_{u}$ an estimate of the average volumetric density of
$U$ particles of the universal cloud, defined at least on a broad spatial domain, much larger than the experimental volume, and with a duration, itself broadly including the time $t$. One supposes that $g_{u}$ is more or less constant in this spatial domain and over this duration. Then, the number, $n$, of particles $U$ in the spatial volume $v$ is the random variable with a probability law defined by $P(n=j)=p_{j}\left(v \cdot g_{u}\right)$.

$v \cdot g_{u}$ is the average value, or mathematical expectation, of the so-defined random variable.

We propose $g_{u} \sim 0.29$ per natural unit of volume, i.e. $g_{u} \sim 2$. $E^{34}$ per cubic meter. We explain where this figure comes from in what follows.

4) Each particle of $U$, of masse $m_{u}$ and speed $v_{u}$, has a kinetic energy $w_{u}=m_{u} \cdot v_{u}^{2} / 2$

Poisson law being associated to a negative exponential, we suppose that the probability density of $v_{u}$ is proportional to $\exp \left(-\frac{v_{u}}{\bar{v}_{u}}\right), \bar{v}_{u}$ being the average value of $v_{u}$.

We do not pretend at all that this hypothesis on the distribution of speeds is the only one compatible with the previous hypothesis on the distribution of the particles' number in a determined volume, but it is the most appealing one. One may note that it gets perceptibly close to Boltzmann analysis thanks to the properties of the exponential function. According to Boltzmann analysis, the probability density of the kinetic energy of a $U$ particle would be proportional to $\exp \left(-\frac{w_{u}}{k \cdot T_{u}}\right)$ with $k$ the Boltzmann constant and $T_{u}$ the temperature in Kelvin degrees of the cloud.

Be $p_{u}$ the probability density of $v_{u}$. with

$$
p^{u}=\frac{1}{\bar{v}_{u}} \cdot e^{-\frac{v_{u}}{\bar{v}_{u}}}
$$

one checks that $\int_{0}^{\infty} v_{u} \cdot p_{u} \cdot \mathrm{d} v_{u}=\bar{v}_{u}$.

Be $\bar{w}_{u}$ the mean value of $w_{u}$, one can also check that $\bar{w}_{u}=\int_{0}^{\infty} w_{u} \cdot p_{u} \cdot \mathrm{d} v_{u}=m_{u} \cdot \bar{v}_{u}^{2}$.

We will estimate the temperature, $T_{u}$, of the cloud by $T_{u}=\bar{w}_{u} / k$.

In what follows, we propose a set of values of $m_{u}$, $\bar{v}_{u}$ and $\bar{w}_{u}$.

We ignore any other characteristic of the $U$ particle. It is possible that the $U$ particle is not elementary, or else that it owns two opposed electrical loads.

In short, the cloud is homogeneous, isotropic, and with a probability density of the absolute speeds proportional to a negative exponential of this absolute speed.

Be two identical particles, for instance two electrons, 
$E_{1}$ and $E_{2}$, in the cloud. $U$ particles knock the electrons, and providing one exactly knows the conditions of a shock and its effects, one can deduct from these shocks the apparent interaction between $E_{1}$ and $E_{2}$. The definition of a shock also needs the definition of the crosssection, similar to the meridian section of a sphere representing a particle but we will soon see that such a definition is quite inadequate.

Whatever the definition of a shock, the symmetries of the system command a certain format for the average expected result. In the present case, the symmetries of the considered system - the universal cloud and two identical particles - command that the interaction between two motionless particles be radial, attractive or repulsive. It is for instance a force $f=x \cdot \frac{r}{r} \cdot r^{y}, x-$ a real positive number, $y$-any real number, $r$ - a vector linking suitably the positions of the particles under the assumption that the speed of these particles is much smaller than the speed of an $U$ particle. Observations show that $x \approx \alpha /(2 \pi)$ and $y \approx-2$, the force being repulsive.

Be now two identical particles except for their electrical load, an electron and a positon. The symmetries of the system and the observations suggest that the interacttion is the attractive force $f=\frac{\alpha}{2 \pi} \cdot \frac{r}{r} \cdot r^{-2}$, equal, in absolute value, to the former one.

Be now two distinct particles, an electron and a proton, $E$ and $P$, in the cloud. The proton has the same electrical load as the positon but it differs from positon regarding other characteristics and one thus expects that interaction be a force different from the previous one. Observations do not exhibit any difference. So, we keep in mind that should a discrepancy exist, it is small, too small to be noticeable.

How can these average results be induced by the shocks of $U$ particles on the two considered particles, i.e. what is the model of particles and shocks able to give birth to these results?

If one attributes to each particle descriptive characteristics such as an unvarying cross section, the conclusion is straightforward-it is impossible to get the obtained results. On the contrary, by revising the concept of inertial mass of a particle, one fairly easily builds the sought mode.

\section{Inertial Mass and Train}

Mach [2] has explained the inadequacy of the mass concept according to Newton's proposals. It is this criticism, together with a recent mathematical result [3] which led us to propose the existence of an universal cloud, not at all as ether in which electromagnetic waves propagate but as a medium liable to confer a mass to any $M$ material particle.

An $M$ particle would be an electrical particle surrounded by a train of $U$ particles. If the train is void, the particle has no mass. The train is modified at every shock with a particle of $U$, either by its composition with one $U$ particle added or removed, or by the arrangement of the $U$ particles within the train, including their absolute orientation. When the train is large, say a hundred of particles or more, it is modified progressively in a way it keeps track of the previous induced characteristics, for instance, the characteristic induced by the presence of another particle in its vicinity. So, the train of a particle, and therefore its mass is not a "ne variatur" attached characteristic to this particle, it is a characteristic mostly attached to this particle but also partly dependent on the recent past story of this particle.

It took us several years to imagine that and get convinced by the huge explanation potential laying in this approach with regard to the interpretation of the most fundamental physical phenomena.

How simple and easy thanks to its simplicity is the concept of mass of a material point according to Newton. To replace it by a mass which depends upon its environment is not much appealing but one has do it because it is the only way to explain observations in a rational way. We discover that the model of Nature is not expressed in the simple mathematical way which we thought and which one always looks for preferentially.

Cross sections mostly depend on the trains of particles. Thus, modeling the shocks may be combined in order for prequantum physics to simultaneously and similarly induce the electromagnetic and gravitational effects, up to the finest detail.

Nota: Observing the hydrogen atom reveals diverse fine details, all rediscovered from prequantum physics. The obvious reason is the following: theses details are described thanks to four quantum numbers. An approximate description of the train of the hydrogen atom, made of a few particles from $U$ can be done with four integer numbers, fairly similar to quantum numbers. The profound reason: quantum mechanics is dominated by the omnipresent discrete character and the quest of results imposed by diverse symmetries, such as the invariance of the gauge. The expected mean results stemming from prequantum physics are also dominated by the discrete character of the universal cloud and its symmetry properties.

Nota: Quantum chromodynamics is also founded on the quest of results imposed by the virtue of certain symmetries. For us, prequantum physics potentially contains quantum chromodynamics but we have not looked for a detailed demonstration of this point. This the reason why we have titled our proposals "A prequantum atomic physics" [1]. 


\section{Explanatory Cross-Sections}

We can now use the "train" concept to define the crosssections suitable to induce electromagnetic and gravitational interactions. We go to the simplest and many other shock models can be imagined.

We have exposed, cf. [1], how to link the trains to a certain descriptive model of the shocks. One still retains the conservation of momentum and the conservation of energy throughout the shock but this still leaves plenty of room for the description, especially because the energy of the train depends on every descriptive detail regarding this train, and with much weight as compared to the kinetic energy variations. This allows us to retain, for a shock, a transfer of momentum of the knocking particle to the $M$ knocked particle. The energy conservation throughout the shock is always compatible with this transfer of momentum.

For a $U$ particle arrowed to meet the train of an $M$ particle, this train may either stop or let it go through depending on the direction of the knocking particle because the $M$ train bears also an orientation which stems from its recent environment. The train may also emit, after the shock and the absorption of the knocking particle in the train, two particles of opposed directions, directions set in relation with the recent past environment.

In short, $M$ particles have a memory which billiard balls have not.

We retain the following description of the diverse cross sections and of their effects:

Shocks may be split into two categories, those which only affect a single particle $M$ and which can be modeled as shocks between two billiard balls, and those which affect two $M$ particles because the speed of the knocking particle of $U$ is more or less aligned on the direction linking the two $M$ particles.

The effects of the first category shocks more or less compensate because their mean effects on long duration become negligible compared to those of the second category which may accumulate without compensation (for instance because of screen effect).

One can therefore limit the examination to the second category shocks.

The average time interval between two successive shocks of a particle $M$ having a cross section $\sigma$ is $\theta=\frac{1}{g_{u} \cdot \sigma \cdot \bar{v}_{u}}$, with $\bar{v}_{u}$ designating the mean absolute speed of the $U$, universal cloud, particles.

One of the effects of a shock against an $M$ particle, of mass $M$ at the time of the shock, by a $U$ particle is the $M$ speed variation $D v, \Delta v=\frac{m_{u}}{m} \cdot v_{u}, v_{u}$ speed of the $U$ referred particle before the shock.

$\mathrm{Be}$ an ordered couple of two particles, proton or elec- tron. The $M_{i}$ particle with $I=1,2$ of $i$ rank is a proton if $j(i)=1$, an electron if $j(i)=-1$. One puts $\delta=j(1) . j(2)$ and one notes that $\delta$ has only two values, 1 and -1 .

Be a particle $U_{k}, k=1,2$, with a speed roughly aligned on $M_{1}$ towards $M_{2}$ direction if $k=1$, on $M_{2}$ towards $M_{1}$ direction if $k=2$.

$\mathrm{Be} \sigma_{i, \delta, k}$ the cross section of the $M_{i}$ particle in regard to the shock with a $U_{k}$ particle.

The eight values of $\sigma_{i, \delta, k}$ are not all distinct. Actually:

1) $\sigma_{i, \delta, k}=\sigma_{3-i, \delta, 3-k}$ by space-time symmetry.

2) $\sigma_{1,1, k}=\sigma_{2,1, k}$ by identity of the $M$ particles.

One proposes, with $0<s_{g} \quad s_{f}$ :

In the case $\delta=-1, \sigma_{i,-1, k}=\sigma_{f}+\sigma_{g}$. The effect is attractive.

In the case $\delta=1, \sigma_{i, l, i}=\sigma_{g}$. The effect is, attractive, small. In the case $\delta=1, \sigma_{i, 1,3-i}=\sigma_{f}+\sigma_{g}=\sigma_{i,-1, k}$. The effect is repulsive, much larger than the previous one, therefore the total combined effect is repulsive.

Be $r$ the vector from $M_{1}$ to $M_{2}$ if $k=1$, from $M_{2}$ to $M_{1}$ if $k=2$ at the time of the shock by the $U_{k}$ particle, taking for granted that the speed of an $U_{k}$ particle is always much larger than the speed of a $M$ particle.

Case $I=k$ : The speed of the $U_{k}$ particle knocking the $M_{k}$ particle is within the cone having for summit the middle of $M_{1}-M_{2}$, and for basis the circle, centered on $M_{k}$, perpendicular to the $M_{1}-M_{2}$ axis, with a ray $=\sqrt{\sigma_{3-k, \delta, 3-k} / \pi}$.

Case $i=3-k$ : The speed of the $U_{k}$ particle knocking the $M_{i}$ particle is, on the one hand, within the cone having for summit the middle of $M_{1}-M_{2}$, and for basis the circle centered on $M_{k}$, perpendicular to $M_{1}-M_{2}$ axis with a ray $=\sqrt{\sigma_{3-k, \delta, k} / \pi}$. and, on the other hand, outside the cone having for summit the middle of $M_{1}-M_{2}$, and for basis the circle centered on $\mathrm{M}_{\mathrm{k}}$, perpendicular to the $M_{1}-$ $M_{2}$ axis, with a ray $=\sqrt{\sigma_{k, \delta, k} / \pi}$.

The mean effect of a shock on the $M_{i}$ particle of mass $m_{i}$ by an $U_{k}$ particle is the speed variation $\Delta v$ of $M_{i}$ with $\Delta v=\frac{m_{u}}{m_{i}} \cdot \frac{r}{\mathrm{r}} \cdot \bar{v}_{u}$.

The average time duration between two successive shocks of an $U_{i}$ particle on a $M_{i}$ particle is:

$$
\tau=\frac{1}{\sigma_{i, \delta, i} \cdot g_{u} \cdot \bar{v}_{u}} \cdot \frac{2 \pi r^{2}}{\sigma_{3-i, \delta, 3-i}}=\frac{2 \pi r^{2}}{\sigma_{i, \delta, i}^{2} \cdot g_{u} \cdot \bar{v}_{u}}
$$

The average time duration between two successive shocks of an $U_{3-i}$ particle on a $M_{i}$ particle is:

$$
\tau=\frac{1}{\sigma_{i, 1,3-i} \cdot g_{u} \cdot \bar{v}_{u}} \cdot \frac{2 \pi r^{2}}{\sigma_{3-i, 1, i}-\sigma_{3-i, 1,3-i}}=\frac{2 \pi r^{2}}{\sigma_{f}^{2} \cdot g_{u} \cdot \bar{v}_{u}} .
$$

This case implies $\delta=1$.

Be $f / r^{2}$ the force, in absolute value, induced by $\sigma_{f} ; f$ is proportional to $\sigma_{f}^{2}$ by screen effect.

$\mathrm{Be} g / r^{2}$ the force, in absolute value, induced by 
$\sigma_{g} ; g$ is proportional to $\sigma_{\mathrm{g}}{ }^{2}$ by screen effect.

Be $f_{a} / r^{2}=\frac{\alpha}{2 \pi r^{2}}$ the electrical force, in absolute value between proton and electron, attractive force. Let us suppose that $f_{r} / r^{2}$, in absolute value, between proton and proton, repulsive force, be weaker, in e in relative value, than $f_{a} / r^{2}$ with $f_{r} / r^{2}=\frac{\alpha \cdot(1-\varepsilon)}{2 \pi r^{2}}$.

By the virtue of the preceding analysis:

$$
f_{a}=f+g ; f_{r}=f-g ; f_{a}-f_{r}=\frac{\alpha \cdot \varepsilon}{2 \pi}=2 \cdot g .
$$

$\mathrm{Be} G m_{A}^{2} / r^{2}$ the universal attraction force between two hydrogen atoms, $m_{A}$ designating the mass of an hydrogen atom.

Prequantum physics simultaneously induces electrical and gravitational under the two conditions that define shocks:

1) $g$ is adjusted on the gravitational force via

$$
g=\frac{f_{a}-f_{r}}{2}=\frac{G \cdot m_{A}^{2}}{4} .
$$

2) $f$ is adjusted on the attractive electrical force via

$$
f=\frac{\alpha}{2 \pi}-\frac{G \cdot m_{A}^{2}}{4} \quad \frac{\alpha}{2 \pi} .
$$

On such bases, the description of the shock of an $U$ particle with respectively, a proton, an electron, an hydrogen atom in terms of average cross section associated to each of these three shocks does not present any difficulty.

From $f$ and $g$, one deduces, by inversion $\sigma_{\mathrm{f}}$ and $\sigma_{\mathrm{g}}$, and from there, all cross sections $\sigma_{\mathrm{i}, \delta, \mathrm{k}}$ with $I=1,2 ; \delta=1,-1$; $k=1,2$.

However, we again underline, as we did for the inertial mass, the new and surprising characteristic of cross sections. A cross section is not a particle characteristic, but a characteristic stemming from the train of this particle, a train which depends on the recent former environment of the particle. We have extensively developed this point [1] regarding the fine prequantum model of the hydrogen atom.

\section{Mass of Neutrons, Protons and Electrons}

Any particle, neutron, proton, electron, is surrounded by a train of $U$ particles which commands its mass and is part of its internal structure. Any particle, neutron, proton, electron, of which the train is void has a nil mass, cf. [1]. The trains of neutrons and protons can certainly be partitioned in diverse quarks. On the contrary, gluons are only apparent particles, bearing statistical information, induced by prequantum physics, with a statute similar to that of a pressure or temperature induced by statistical mechanics founded on atomistic. Idem for the photon.

\subsection{Numerical Values Relative to the $U$ Particles: Average Cross Sections of Protons, Electrons, Neutrons, Hydrogen Atoms}

The main descriptive features of prequantum physics are, on the one hand, the $m_{u}$ mass of a particle $U$, the mean absolute speed $\bar{v}_{u}$ of the $U$ particles, the average volumetric density $g_{u}$ of particles of the $U$ universal cloud and, on the other hand, a reduced set of cross sections of protons, electrons, hydrogen atoms.

These diverse cross sections are noted as follows:

$\sigma_{P}, \sigma_{E}$, respective average cross section, all shocks confounded, of the proton and the electron. One retains $\sigma_{E}=\sigma_{P}$

$\sigma_{H}$ average cross section, all shocks confounded, of each hydrogen atom of a couple of two hydrogen atoms.

We have presented [1] the relations between these characteristics and their values to satisfy these relations. We only recall them as follows:

$$
\begin{gathered}
m_{u} \cdot \bar{v}_{u}=g_{u} \cdot \sigma_{E} \\
\frac{1}{2} \cdot m_{u} \cdot \bar{v}_{u}^{2}=1-\frac{\alpha^{2}}{2} \\
\sigma_{E}^{2}=\frac{1}{2 \cdot \pi \cdot g_{u} \cdot \bar{v}_{u}} \\
G=\frac{m_{u} \cdot g_{u} \cdot \sigma_{H}^{2} \cdot \bar{v}_{u}^{2}}{\pi \cdot m_{H}^{2}} \\
\sigma_{E}=\frac{\sigma_{H}}{m_{N}} \cdot \sqrt{\frac{\alpha}{2 \cdot \pi \cdot G}}
\end{gathered}
$$

$m_{H}$ designates the mass of the hydrogen atom in its fundamental state.

The solution of this system of equations in natural units is:

Cross sections: $s_{E}=s_{P}=8.0 E^{-2} ; s_{H}=7.2 E^{-20}$.

Inertial mass of the $U$ particle: $m_{u}=2.6 E^{4}$.

Average volumetric density of $U$ particles in the cloud: $g_{u}=0.29$.

Absolute average speed of the $U$ particles: $\bar{v}_{u}=87$. Reminder: the natural unit of speed is $c$, speed of light.

From this solution, one deduces the following characters, in natural units:

Mean energy of an $U$ particle, $\bar{w}_{u}=m_{u} \cdot \bar{v}_{u}^{2} 2$.

Temperature $T_{u}$ attributed to the cloud via $k \cdot T_{u}=\bar{w}_{u}$, $T_{u}=12 . E^{9}$ Kelvin degrees $\sigma_{g}=1.8 E^{-20} ; \sigma_{f}=8.0 E^{-2}$.

Mean time interval $\theta_{E}$ between successive shocks of $U$ and a charged particle, proton or electron, of a protonelectron couple, $\theta_{E}=\frac{1}{g_{u} \cdot \sigma_{E} \cdot v_{u}} \quad 0.5$, i.e. half the 
retained natural unit of time. (Reminder: the retained time unit is $\frac{h}{m \cdot c^{2}}$ ).

Mean time interval $\theta_{H}$ between two successive apparent shocks of $U$ on an hydrogen atom of a couple of hydrogen atoms $\theta_{H}=\frac{1}{g_{u} \cdot \sigma_{H} \cdot v_{u}} \quad 5.6 E^{17}$, of the order of magnitude of one ten thousandth of second $(0.0001$ second).

\subsection{Trains of Neutrons and Protons}

The internal structure of neutrons and protons is built on quarks and gluons. According to prequantum physics, the neutron and the proton each have a train made of more than one hundred of $U$ particles around a few electrical charges. The research of stable-according to prequantum physics - dynamic configurations of such complex systems as a set of protons and neutrons, exceeds widely can be today achieved by mathematic tools.

Gluons, as the photon, would only be bearers of information, stemming from the underlying statistical mechanics. Quarks and gluons are the pieces of an approximate model, by statistical characters, of the most stable dynamic configurations, model independent from the detail of particle movements at the prequantum level. It is a model akin to Boltzmann analysis in the sense that it is mostly guided by symmetry considerations.

For what concerns the gravitational effects between uncharged material bodies, as one ones observes the in the solar system, it seems that each neutron as an effect comparable to that of an hydrogen atom, uncharged particle with a similar mass.

\section{Waves and photon}

The description of the electromagnetic waves by Maxwell equations is both precise and sufficient for many practical applications based on emitters and receptors.

However, when it was born in the 19th century, it was not considered complete, because a wave was associated to a propagation medium whereas the propagation medium of electromagnetic waves was unknown. It was named "ether" and attempts were made to put into evidence but the experiences conducted for that purpose gave a result opposite to the expectations. There was therefore a need of imagining a theory without ether, which led to modify the space and time structures as space and time are at the heart of any wave motion.

With this new conception of space and time, ether may be discarded but per contra, a photon-particle able to move in vacuum-must be introduced, a mysterious photon since it appears in certain experiences as endowed with the properties of a wave. In reality, all theses successive difficulties and resolution attempts are a clue that the electromagnetic wave model remains incomplete. In particular, we do not know why these waves are transverse and not longitudinal as air-propagating sound waves.

The transverse character is important but, for what concerns the practical applications, it little matters to know why the wave is transverse.

Prequantum physics suggest the following answer, stemming quasi-directly from the concept of train with variable composition: train of any $M$ particle, variable because of certain shocks of $U$ particles on $M$ particles.

One knows that electromagnetic waves are fathered by certain alternates movements of electrical charges, for instance by a rotating electrical dipole. Let us consider a pair of particles of opposite charges, a proton and an electron, fairly close to each other.

Nota: We say a pair electron-proton and not an hydrogen atom because this pair may belong to any atom or any body such as the sun in which most of hydrogen atoms are disintegrated.

The pair is knocked incessantly by $U$ particles. Shocks are not elastic and the train of the pair, i.e. the gathering of the trains of the proton and electron, is modified at every shock. Among these shocks, those which affect one of the particles of the pair without affecting the other one have effects which, statistically, roughly compensate because of the disorder of the $U$ cloud, and which do not affect the isotropic character of the cloud. On the contrary, the shocks of any $U$ particle having a speed direction close to that of the dipole, have effects on both particles, a direct effect for one and a screen effect for the other one, effects which are cumulative because of the absence of statistical compensation. The system characteristics are such that, over a brief time interval, several shocks may happen on the proton and electron particles in the course of their small, each to other relative, displacement. These effects break isotropy off.

The train of a pair, because of the impacts of these last shocks, varies incessantly in plus or minus a $U$ particle, and for a minus variation, the pair seems to emit two $U$ particles because of the shock impact. Let us admit, as a property of the trains, that, under the effect of this said mixed shock, two $U$ particles, noted $U_{1}$ and $U_{2}$, are emitted, one in the $K$ direction, the other one in the $-K$ direction, $K$ being the orthogonal direction to the plan of the electron relative trajectory, plan defined by the proton and the displacement of the electron relative to the proton. Let us also admit, as a property of the train, that the speed of the two emitted particles is $c$ and that the emission point is the relative particle, confounded roughly with the electron.

A series of a few $U_{1}$ and $U_{2}$ particles own the character of a transverse wave propagating at $c$ speed. The wave is 
transverse because of the movement of the two particles compared to the propagation speed.

One needs to note 1) that the $c$ wave speed is different from that $v_{u}$ of the $U$ knocking particle which is at the origin of the wave; 2 ) that the wave unfolds symmetrically from its origin; 3 ) that the wave may be emitted in any direction, the osculating plan of the trajectory being whatever; 4) the wave frequency is the inverse of the time interval $D t$ between two successive mixed shocks, and that this time interval varies, on the one hand as $1 / r^{2}, r$ being the distance between two charged particles, and on the other hand as $1 / s_{P}^{2}$, the cross section $\sigma_{P}$ depending of a train which gets larger according to the temperature of the considered particles; 5) that the wave energy, the flux of the Poynting vector is $m_{u} \cdot c^{2} / D t$, natural units; 6) that the theory of the radiative emission of black body may be got back on these bases ${ }^{2}$.

The train concept, for explaining the transverse character of the wave, is essential, the sound wave is longitudinal, and an ether, playing for electromagnetic waves the role of air for the propagation of sound waves, cannot, in any way, explain electromagnetic waves. The universal cloud is an ether, in the sense that electromagnetic waves are propagated by local deformation of that medium, but the origin of this deformation goes through the concept of train and of variable mass. A local deformation of this discrete medium is a local break of the isotropic character, the existence of a few squeezed together trajectories of $U$ particles roughly in the same direction.

The eye is light detector, it is knocked by successive $U_{\mathrm{t}}$ particles which are low speed particles (speed $c$ compared to the $v_{u}$ speeds). It does not at all detect a stream of particles, in the very same way as an eardrum does not at all detect the shocks of oxygen and nitrogen atoms in a noiseless space, the eye only detects transverse oscillations, i.e. series of $U_{t}$ particles with nearly parallel tra-

\footnotetext{
${ }^{2}$ The radiance of the sun is sometimes presented as the emission of photons stemming from the fusion reactions of hydrogen and helium in the central part of sun, followed by a complex progression of these photons up to the surface. According to our proposals, the explanation of the radiance would be slightly different: the exothermic fusion reactions in the central part would maintain a temperature gradient between this region and the surface, i.e. a gradient of average speed of proton and electron particles and a gradient of trains composition because the trains of these particles depend on their speed. The variation of the trains is precisely what limits the speed of these particles below the speed $c$. The radiance would mostly originate from the surface zone of the sun by reduction of the trains, always by pairs of emitted $U$ particles always emitted at speed $c$ towards two opposite directions. The distribution of distances between protons and electrons would be such as the emission be close to that of a black body at the surface temperature. In short, the radiated energy truly comes of the fusion exothermic reactions but the radiance, said emission of photons, is a phenomenon passing through the growth of the trains in the sun central part and their diminution in the surface zone. The $U$ particles emitted by the trains are not photons, statistical fictitious particles, but slow speed, i.e. at $c$ speed, particles. This process explains the transverse character of the waves whereas a photon emissions from the central part cannot explain any transverse character of the electromagnetic wave.
}

jectories. The eye differentiates these oscillations according to their color, i.e. their frequency, in the same exact way, the eardrum differentiates sounds according to their height.

The photon, by the direction of its speed, by its frequency (or energy), by its spin, is bearing statistical information, namely the average characteristics of the wave and of the $U_{1}$ and $U_{2}$ particles. As a bearer of information, it can model a wave as well as a particle. It is neither one nor the other, his statute is that of a temperature. It is observable in a very same way as a temperature is observable.

We have presented [1], regarding the new model of the hydrogen atom, how the photon appears a bearer of statistical information stemming from prequantum physics. It is precisely because the photon has this statute that it may qualify, depending on the needs, either a wave, or a particle. Photon, gravitons, gluons, and other interaction particles, have no more a physical reality than a temperature, but, evidently, this particles are observable and measurable, exactly as a temperature is observable and measurable.

Everything we have exposed regarding electromagnetic waves might possibly be transposed to gravitational waves since prequantum physics only introduces differences through the role of the nature of particles, charged or not. However, this transposition can only reveal gravitational waves of very low frequency, undetectable unless for huge gatherings induced by extraordinary movements of celestial bodies, such as pulsars.

One does not imagine practical applications of gravitational waves. As such, they become scientific imagination, which, with cosmology, leads to take the place called by the inexhaustible curiosity of the well informed branch of mankind.

\section{Scientific Modesty}

Prequantum physics make quantum relativistic mechanics, i.e. the quantum theory of fields and the algorithms of perturbative expansion coupled with the renormalization, appear as stemming from a statistical mechanics. It is a result which seem to us, without practical interest, but philosophically significant, because it reestablishes determinism. It may also re-orientate researches in theoretical physics, always in process and always unfruitful since more than thirty years now.

The limits of knowledge, like time and space, have retained the attention of the greatest thinkers.

Pascal says that in the following manner: Science relates to the links we can establish among measurements of measurable phenomena. The essence of phenomena, measured or not, escaped us.

Kant specifies about time. A good chronometer measures time but to say that there exists a universal time is to admit that the concept of time is given to Man by nature. 
Mach underlines the deficiency of the mass definition in Newton mechanics. Wanting to keep an achieved science, one has, in fact hidden the problem without finding a solution. Today, there is no more a solution but the problem has been forgotten.

Poincaré, mathematician, physicist and philosopher, knows all the weaknesses of building a scientific physics and summarizes as follows: Science is convenient and this is why we believe that there is a little truth in science. It is the pessimism of intelligence but Poincaré, overcoming the part of combined doubt and attraction so widespread in the mathematicians towards physicists, nevertheless invites the scientist to research truth, the only research it should undertake.

\section{REFERENCES}

[1] R. L. Charreton, “A Prequantum Atomic Physics,” 2011. http://perso.numericable.fr/raoul.charreton/

[2] E. Mach, "Die Mechanik in Ihrer Entwickelung: Historisch-Kritisch Dargestellt (Mechanics, Historical and Critical Lecture of Its Development)," F. A. Brockhaus Leipzig, 1883.

[3] R. L. Charreton, "A Law Limit for Random Walks with Applications to Physics," Minutes of the French Academy of Science (Comptes Rendus de l'Académie des Sciences), Elsevier, Paris, 2007, pp. 699-703. 